\title{
The optimal projection equations for reduced-order, discrete-time state estimation for linear systems with multiplicative white noise
}

\author{
Wassim M. HADDAD \\ Mechanical Engineering Department, Florida Institute of Technology, Melbourne, FL 32901, USA \\ Dennis S. BERNSTEIN * \\ Harris Corporation, Government Aerospace Systems Division, MS 22/4842, Melbourne, FL 32902, USA
}

Received 2 July 1986

\begin{abstract}
The optimal projection equations obtained in [2,3] for reduced-order, discrete-time state estimation are generalized to include the effects of state- and measurement-dependent noise to provide a model of parameter uncertainty. In contrast to the single matrix Riccati equation arising in the full-order (Kalman filter) case, the optimal steady-state reduced-order discrete-time estimator is characterized by three matrix equations (one modified Riccati equation and two modified Lyapunov equations) coupled by both an oblique projection and stochastic effects.
\end{abstract}

Keywords: Reduced-order Kalman filter, Robust estimation.

\section{Introduction}

In a recent series of papers [1-3] it has been shown that the first-order necessary conditions for optimal continuous and discrete-time reduced-order state-estimation can be transformed into coupled systems of three matrix equations (one modified Riccati equation and two modified Lyapunov equations). The coupling is due to the presence of an oblique projection (idempotent matrix) which arises as a rigorous consequence of the stationarity conditions. This formulation provides a direct generalization of the classical steady-state Kalman filter theory. Specifically, in the full-order case, the projection becomes the identity matrix, the additional two modified Lyapunov equations drop out, and the remaining modified Riccati equation reduces to the standard observer Riccati equation for the Kalman filter expression. Related results in reduced-order estimator design can be found in [4-17].

An additional extension of classical state estimation involves the inclusion of state- and measurementdependent disturbances [18-24]. One motivation for such a model is to design estimators which are desensitized, i.e., robustified, to actual parameter variations [25-31]. For the continuous-time control problem this has been justified in [32-38].

As shown in [36] for the continuous-time case, applying the optimal projection approach to the multiplicative white noise model yields an extended formulation of the optimality conditions for reducedorder state estimation. Specifically, the system of three matrix equations characterizing the optimal estimator are now coupled by both the oblique projection and stochastic effects.

* Supported in part by the Air Force Office of Scientific Research under contract F49620-86-C-0002. 
The purpose of the present paper is to provide a self-contained derivation of the optimality conditions for reduced-order state estimation in the presence of both state- and measurement-dependent white noise in the discrete-time case. The goal of the development is to present the optimality conditions in a clear, concise manner to facilitate the development of numerical algorithms for practical application.

\section{Notation and definitions}

$\mathbb{R}, \mathbb{R}^{r \times s}, \mathbb{R}^{r}, \mathrm{E}$ real numbers, $r \times s$ real matrices, $\mathbb{R}^{r \times 1}$, expectation.

$I_{n},()^{\mathrm{T}}, \otimes \quad n \times n$ identity, transpose, Kronecker product [39].

$\tau_{\perp} \quad I_{n}-\tau, \tau \in \mathbb{R}^{n \times n}$.

$n, m, l, n_{\mathrm{e}}, q$ positive integers, $1 \leq n_{\mathrm{e}} \leq n$.

$x, x_{\mathrm{e}} \quad n, n_{\mathrm{e}}$-dimensional vectors.

$y, y_{\mathrm{e}} \quad l, q$-dimensional vectors.

$A, A_{i} ; C, C_{i} \quad n \times n$ matrices, $l \times n$ matrices, $i=1, \ldots, p$.

$A_{\mathrm{e}}, B_{\mathrm{e}}, C_{\mathrm{e}}, D_{\mathrm{e}} n_{\mathrm{e}} \times n_{\mathrm{e}}, n_{\mathrm{e}} \times l, q \times n_{\mathrm{e}}, q \times l$ matrices.

$k \quad$ discrete-time index $1,2,3, \ldots$

$v_{i}(k) \quad$ unit variance white noise, $i=1, \ldots, p$.

$w_{1}(k), w_{2}(k) \quad n$-dimensional, l-dimensional white noise processes.

$V_{1} \quad n \times n$ nonnegative-definite covariance of $w_{1}(k)$.

$V_{2} \quad l \times l$ positive-definite covariance of $w_{2}(k)$.

$V_{12} \quad n \times l$ cross-covariance of $w_{1}(k), w_{2}(k)$.

$R \quad q \times q$ positive-definite matrix.

$L \quad q \times n$ matrix.

$$
\begin{aligned}
& \tilde{A}=\left[\begin{array}{cc}
A & 0 \\
B_{\mathrm{e}} C & A_{\mathrm{e}}
\end{array}\right], \quad \tilde{A_{i}}=\left[\begin{array}{cc}
A_{i} & 0 \\
B_{\mathrm{e}} C_{i} & 0
\end{array}\right], \quad i=1, \ldots, p, \\
& \tilde{w}(k)=\left[\begin{array}{c}
w_{1}(k) \\
B_{\mathrm{e}} w_{2}(k)
\end{array}\right], \quad \tilde{V}=\left[\begin{array}{cc}
V_{1} & V_{12} B_{\mathrm{e}}^{\mathrm{T}} \\
B_{\mathrm{e}} V_{12}^{\mathrm{T}} & B_{\mathrm{e}} V_{2} B_{\mathrm{e}}^{\mathrm{T}}
\end{array}\right], \\
& \tilde{R}=\left[\begin{array}{cc}
L^{\mathrm{T}} R L-L^{\mathrm{T}} R D_{\mathrm{e}} C-C^{\mathrm{T}} D_{\mathrm{e}}^{\mathrm{T}} R L+C^{\mathrm{T}} D_{\mathrm{e}}^{\mathrm{T}} R D_{\mathrm{e}} C+\sum_{i=1}^{p} C_{i}^{\mathrm{T}} D_{\mathrm{e}}^{\mathrm{T}} R D_{\mathrm{e}} C_{i} & -L^{\mathrm{T}} R C_{\mathrm{e}}+C^{\mathrm{T}} D_{\mathrm{e}}^{\mathrm{T}} R C_{\mathrm{e}} \\
-C_{\mathrm{e}}^{\mathrm{T}} R L+C_{\mathrm{e}}^{\mathrm{T}} R D_{\mathrm{e}} C & C_{\mathrm{e}}^{\mathrm{T}} R C_{\mathrm{e}}
\end{array}\right] .
\end{aligned}
$$

$Z_{(i, j)} \quad(i, j)$ element of matrix $Z$.

$\rho(Z) \quad$ rank of matrix $Z$.

$\operatorname{tr} Z \quad$ trace of a square matrix $\dot{Z}$.

$E_{i} \quad$ square matrix with unity in the $(i, i)$ position and zeros elsewhere.

$\pi_{i}(\psi) \quad \psi E_{i} \psi^{-1}$ (unit-rank eigenprojection).

$\mathscr{N}(Z), \mathscr{R}(Z)$ null space, range of matrix $Z$.

An asymptotically stable matrix is a matrix with eigenvalues in the open unit disk; a nonnegative-definite matrix is a symmetric matrix with nonnegative eigenvalues; and a positive-definite matrix is a symmetric matrix with positive eigenvalues.

For arbitrary $n \times n, Q, \hat{Q}, \tau$, define

$$
\begin{array}{ll}
V_{2 \mathrm{~s}} \triangleq V_{2}+C Q C^{\mathrm{T}}+\sum_{i=1}^{p} C_{i}\left(Q+\tau \hat{Q} \tau^{\mathrm{T}}\right) C_{i}^{\mathrm{T}}, & Q_{\mathrm{s}} \triangleq A Q C^{\mathrm{T}}+V_{12}+\sum_{i=1}^{p} A_{i}\left(Q+\tau \hat{\hat{Q}} \tau^{\mathrm{T}}\right) C_{i}^{\mathrm{T}}, \\
\hat{V}_{2 \mathrm{~s}} \triangleq V_{2}+C Q C^{\mathrm{T}}+\sum_{i=1}^{p} C_{i}(Q+\hat{Q}) C_{i}^{\mathrm{T}}, & \hat{Q}_{\mathrm{s}} \triangleq A Q C^{\mathrm{T}}+V_{12}+\sum_{i=1}^{p} A_{i}(Q+\hat{Q}) C_{i}^{\mathrm{T}} .
\end{array}
$$




\section{Problem statement and main theorem}

Reduced-Order State-Estimation Problem. Given the $n$-th-order observed system

$$
\begin{aligned}
& x(k+1)=\left(A+\sum_{i=1}^{p} v_{i}(k) A_{i}\right) x(k)+w_{1}(k), \\
& y(k)=\left(C+\sum_{i=1}^{p} v_{i}(k) C_{i}\right) x(k)+w_{2}(k),
\end{aligned}
$$

design an $n_{\mathrm{e}^{- \text {th }}}$ reduced-order state estimator

$$
\begin{aligned}
& x_{\mathrm{e}}(k+1)=A_{\mathrm{e}} x_{\mathrm{e}}(k)+B_{\mathrm{e}} y(k), \\
& y_{\mathrm{e}}(k)=C_{\mathrm{e}} x_{\mathrm{e}}(k)+D_{\mathrm{e}} y(k),
\end{aligned}
$$

which minimizes the state-estimation error criterion

$$
J\left(A_{\mathrm{e}}, B_{\mathrm{e}}, C_{\mathrm{e}}, D_{\mathrm{e}}\right) \triangleq \lim _{k \rightarrow \infty} \mathrm{E}\left[L x(k)-y_{\mathrm{e}}(k)\right]^{\mathrm{T}} R\left[L x(k)-y_{\mathrm{e}}(k)\right] .
$$

In this formulation the matrix $L$ identifies the states, or linear combinations of states, whose estimates are desired. The order $n_{\mathrm{e}}$ of the estimator state $x_{\mathrm{e}}$ is determined by implementation constraints, i.e., by the computing capability available for realizing (3.3), (3.4) in real time. Note that the feedthrough term $D_{\mathrm{e}}$ permits the utilization of a static least squares estimator in conjunction with the dynamic estimator $\left(A_{\mathrm{e}}, B_{\mathrm{e}}, C_{\mathrm{e}}\right.$ ). Thus, the goal of the Reduced-Order State-Estimation Problem is to design an estimator of given order that yields quadratically optimal (least squares) estimates of specified linear combinations of states.

To guarantee that $J$ is finite assume that $A$ is asymptotically stable and consider the set of asymptotically stable reduced-order (i.e., fixed-order) estimators

$$
\mathscr{P} \triangleq\left\{\left(A_{\mathrm{e}}, B_{\mathrm{e}}, C_{\mathrm{e}}, D_{\mathrm{e}}\right): A_{\mathrm{e}} \text { is asymptotically stable }\right\} .
$$

Since the value of $J$ is independent of the internal realization of the transfer function corresponding to (3.3) and (3.4), without loss of generality we further restrict our attention to the set of admissible estimators

$$
\mathscr{S}^{+} \triangleq\left\{\left(A_{\mathrm{e}}, B_{\mathrm{e}}, C_{\mathrm{e}}, D_{\mathrm{e}}\right) \in \mathscr{S}:\left(A_{\mathrm{e}}, B_{\mathrm{e}}\right) \text { is controllable and }\left(A_{\mathrm{e}}, C_{\mathrm{e}}\right) \text { is observable }\right\} .
$$

The following factorization lemma is needed for the statement of the main result.

Lemma 3.1. Let $\tau \in \mathbb{R}^{n \times n}$. Then

$$
\begin{aligned}
& \tau^{2}=\tau, \\
& \rho(\tau)=n_{\mathrm{e}},
\end{aligned}
$$

if and only if there exist $G, \Gamma \in \mathbb{R}^{n_{\mathrm{e}} \times n}$ such that

$$
\begin{aligned}
& G^{\mathbf{T}} \Gamma=\tau, \\
& \Gamma G^{\mathrm{T}}=I_{n_{\mathrm{e}}} .
\end{aligned}
$$

Furthermore, $G$ and $\Gamma$ are unique to a change of basis in $\mathbb{R}^{n_{\mathrm{e}}}$. 
Proof. See [3].

For convenience, call $G$ and $\Gamma$ satisfying (3.8) and (3.9) a projective factorization of $\tau$. Furthermore, for $n \times n$ nonnegative-definite matrices $\hat{Q}$ and $\hat{P}$, define the set of contragrediently diagonalizing transformations

$$
\mathscr{D}(\hat{Q}, \hat{P}) \triangleq\left\{\psi \in \mathbb{R}^{n \times n}: \psi^{-1} \hat{Q} \psi^{-\mathrm{T}} \text { and } \psi^{\mathrm{T}} \hat{P} \psi \text { are diagonal }\right\} .
$$

It follows from Theorem 6.2.5, p. 123 of [40], that $\mathscr{D}(\hat{Q} ; \hat{P})$ is always nonempty. This set does not, however, have a unique element since basis rearrangements and sign transpositions may be incorporated into $\psi$. Further nonuniqueness arises if $\hat{Q} \hat{P}$ has repeated eigenvalues.

Theorem 3.1. Suppose $A$ is asymptotically stable and $\left(A_{\mathrm{e}}, B_{\mathrm{e}}, C_{\mathrm{e}}, D_{\mathrm{e}}\right) \in \mathscr{S}^{+}$solves the Optimal ReducedOrder State-Estimation Problem. Then there exist $n \times n$ nonnegative-definite matrices $Q, \hat{Q}$ and $\hat{P}$ such that $A_{\mathrm{e}}, B_{\mathrm{e}}, C_{\mathrm{e}}$ and $D_{\mathrm{e}}$ are given by

$$
\begin{aligned}
& A_{\mathrm{e}}=\Gamma\left(A-Q_{\mathrm{s}} V_{2 \mathrm{~s}}^{-1} C\right) G^{\mathrm{T}}, \\
& B_{\mathrm{e}}=\Gamma Q_{\mathrm{s}} V_{2 \mathrm{~s}}^{-1}, \\
& C_{\mathrm{e}}=\left(L-D_{\mathrm{e}} C\right) G^{\mathrm{T}}, \\
& D_{\mathrm{e}}=L Q C^{\mathrm{T}} V_{2 \mathrm{~s}}^{-1},
\end{aligned}
$$

and such that $Q, \hat{Q}$ and $\hat{P}$ satisfy

$$
\begin{aligned}
& Q=A Q A^{\mathrm{T}}+\sum_{i=1}^{p} A_{i}\left(Q+\tau \hat{Q} \tau^{\mathrm{T}}\right) A_{i}^{\mathrm{T}}+V_{1}-Q_{\mathrm{s}} V_{2 \mathrm{~s}}^{-1} Q_{\mathrm{s}}^{\mathrm{T}}+\tau_{\perp} \hat{Q} \tau_{\perp}^{\mathrm{T}}, \\
& \hat{Q}=A \tau \hat{Q} \tau^{\mathrm{T}} A^{\mathrm{T}}+Q_{\mathrm{s}} V_{2 \mathrm{~s}}^{-1} Q_{\mathrm{s}}^{\mathrm{T}}, \\
& \hat{P}=\left(A-Q_{\mathrm{s}} V_{2 \mathrm{~s}}^{-1} C\right)^{\mathrm{T}} \tau^{\mathrm{T}} \hat{P} \tau\left(A-Q_{\mathrm{s}} V_{2 \mathrm{~s}}^{-1} C\right)+\left(L-D_{\mathrm{e}} C\right)^{\mathrm{T}} R\left(L-D_{\mathrm{e}} C\right),
\end{aligned}
$$

where

$$
\tau \triangleq \sum_{i=1}^{n_{\mathrm{e}}} \pi_{i}(\psi)
$$

for some $\psi \in \mathscr{D}(\hat{Q}, \hat{P})$ such that $\left(\psi^{-1} \hat{Q} \hat{P} \psi\right)_{(i, i)} \neq 0, i=1, \ldots, n_{\mathrm{e}}$, and some projective factorization $G, \Gamma$ of $\tau$. Furthermore, the minimal cost is given by

$$
J\left(A_{\mathrm{e}}, B_{\mathrm{e}}, C_{\mathrm{e}}, D_{\mathrm{e}}\right)=\operatorname{tr}\left[\left(L Q L^{\mathrm{T}}-D_{\mathrm{e}} V_{2 \mathrm{~s}} D_{\mathrm{e}}^{\mathrm{T}}\right) R\right] .
$$

Remark 3.1. It is useful to note that (3.10) can be replaced by

$$
A_{\mathrm{e}}=\Gamma A G^{\mathrm{T}}-B_{\mathrm{e}} C G^{\mathrm{T}} \text {. }
$$

Remark 3.2. Because of (3.9) the $n \times n$ matrix $\tau$ which couples the three equations (3.14)-(3.16) is idempotent, i.e., $\tau^{2}=\tau$. In general, this 'optimal projection' is an oblique projection (as opposed to an orthogonal projection) since it is not necessarily symmetric. It should be stressed that the form of the optimal reduced-order estimator (3.10)-(3.13) is a direct consequence of optimality and not the result of an a priori assumption on the structure of the reduced-order estimator.

Remark 3.3. To specialize the result to the strictly proper (no feedthrough) case, merely ignore (3.13) and set $D_{\mathrm{e}}=0$ wherever it appears. 
Remark 3.4. Replacing $x_{e}$ by $S x_{e}$, where $S$ is invertible, yields the 'equivalent' estimator $\left(S A_{\mathrm{e}} S^{-1}, S B_{\mathrm{e}}, C_{\mathrm{e}} S^{-1}, D_{\mathrm{e}}\right)$ with $J\left(S A_{\mathrm{e}} S^{-1}, S B_{\mathrm{e}}, C_{\mathrm{e}} S^{-1}, D_{\mathrm{e}}\right)=J\left(A_{\mathrm{e}}, B_{\mathrm{e}}, C_{\mathrm{e}}, D_{\mathrm{e}}\right)$. Note that transformation of the estimator state basis corresponds to the alternative factorization $\tau=\left(S^{-\mathrm{T}} G\right)^{\mathrm{T}}(S T)$.

Remark 3.5. Note that for the optimal values of $A_{\mathrm{e}}$ and $B_{\mathrm{e}}$ the estimator dynamics (3.3) assume the usual observer form

$$
x_{\mathrm{e}}(k+1)=\Gamma A G^{\mathbf{T}} x_{\mathrm{e}}+\Gamma Q_{\mathrm{s}} V_{2 \mathrm{~s}}^{-1}\left(y-C G^{\mathbf{T}} x_{\mathrm{e}}\right) .
$$

By introducing the quasi-full-state estimate $\hat{x} \triangleq G^{\mathrm{T}} x_{\mathrm{e}} \in \mathbb{R}^{n}$ so that $\tau \hat{x}=\hat{x}$ and $x_{\mathrm{e}}=\Gamma \hat{x} \in \mathbb{R}^{n_{\mathrm{e}}},(3.19)$ can be written as

$$
\hat{x}(k+1)=\tau A \tau \hat{x}+\tau Q_{\mathrm{s}} V_{2 \mathrm{~s}}^{-1}(y-C \hat{x}) .
$$

Although the implemented estimator (3.19) has the state $x_{\mathrm{e}} \in \mathbb{R}^{n_{\mathrm{e}}},(3.19)$ can be viewed as a quasi-full-order estimator whose geometric structure is entirely dictated by the projection $\tau$ and the stochastic effects. Specifically, error inputs $Q_{\mathrm{s}} V_{2 \mathrm{~s}}^{-1}(y-C \hat{x})$ are annihilated unless they are contained in $[\mathscr{N}(\tau)]^{\perp}=\mathscr{R}\left(\tau^{\mathrm{T}}\right)$. Hence, the observation subspace of the estimator is precisely $\mathscr{R}\left(\tau^{\mathrm{T}}\right)$.

Specializing Theorem 3.1 to the noise-free case $A_{i}=0, C_{i}=0, i=1, \ldots, p$, yields Theorem 2.2 of $[2,3]$. Alternatively, specializing Theorem 3.1 to the full-order case $n_{e}=n$ reveals that the Lyapunov equation for $\hat{P}$ is superfluous. In this case it follows from Remark 3.4 that $G=\Gamma=I_{n}$ without loss of generality.

Corollary 3.1. Assume $n_{\mathrm{e}}=n, A$ is asymptotically stable and $\left(A_{\mathrm{e}}, B_{\mathrm{e}}, C_{\mathrm{e}}, D_{\mathrm{e}}\right) \in \mathscr{S}^{+}$solves the Optimal Full-Order State-Estimation Problem. Then there exist $n \times n$ nonnegative-definite matrices $Q$ and $\hat{Q}$ such that $A_{\mathrm{e}}, B_{\mathrm{e}}, C_{\mathrm{e}}$ and $D_{\mathrm{e}}$ are given by

$$
\begin{aligned}
& A_{\mathrm{e}}=A-\hat{Q}_{\mathrm{s}} \hat{V}_{2 \mathrm{~s}}^{-1} C, \\
& B_{\mathrm{e}}=\hat{Q}_{\mathrm{s}} \hat{V}_{2 \mathrm{~s}}^{-1}, \\
& C_{\mathrm{e}}=L-D_{\mathrm{e}} C, \\
& D_{\mathrm{e}}=L Q C^{\mathrm{T}} \hat{V}_{2 \mathrm{~s}}^{-1},
\end{aligned}
$$

and such that $Q$ and $\hat{Q}$ satisfy

$$
\begin{aligned}
& Q=A Q A^{\mathrm{T}}+\sum_{i=1}^{p} A_{i}(Q+\hat{Q}) A_{i}^{\mathrm{T}}+V_{1}-\hat{Q}_{\mathrm{s}} \hat{V}_{2 \mathrm{~s}}^{-1} \hat{Q}_{\mathrm{s}}^{\mathrm{T}}, \\
& \hat{Q}=A \hat{Q} A^{\mathrm{T}}+\hat{Q}_{\mathrm{s}} \hat{V}_{2 \mathrm{~s}}^{-1} \hat{Q}_{\mathrm{s}}^{\mathrm{T}} .
\end{aligned}
$$

Furthermore, the minimal cost is given by

$$
J\left(A_{\mathrm{e}}, B_{\mathrm{e}}, C_{\mathrm{e}}, D_{\mathrm{e}}\right)=\operatorname{tr}\left[\left(L Q L^{\mathrm{T}}-D_{\mathrm{e}} \hat{V}_{2 \mathrm{~s}} D_{\mathrm{e}}^{\mathrm{T}}\right) R\right] .
$$

Remark 3.6. To recover the standard Kalman filter result from Corollary 3.1 set $A_{i}=0, C_{i}=0$, $i=1, \ldots, p$, so that (3.25) and (3.26) are decoupled and (3.26) is superfluous. Since the standard Kalman filter is strictly proper, set $D_{\mathrm{e}}=0$ as in Remark 3.3.

\section{Proof of the main theorem}

Using the notation of Section 2 the augmented system (3.1) and (3.3) can be written as

$$
\tilde{x}(k+1)=\left(\tilde{A}+\sum_{i=1}^{p} v_{i}(k) \tilde{A_{i}}\right) \tilde{x}(k)+\tilde{w}(k),
$$


where $\tilde{x}(k) \triangleq\left[x^{\mathrm{T}}(k), x_{\mathrm{e}}^{\mathrm{T}}(k)\right]^{\mathrm{T}}$. To analyze (4.1) it is useful to define the second-moment matrix

$$
\tilde{Q}(k)=\mathrm{E}\left[\tilde{x}(k) \tilde{x}^{\mathrm{T}}(k)\right] .
$$

It follows from (4.1) and (4.2) that $\tilde{Q}(k)$ must satisfy

$$
\tilde{Q}(k+1)=\tilde{A} \tilde{Q}(k) \tilde{A}^{\mathrm{T}}+\sum_{i=1}^{p} \tilde{A}_{i} \tilde{Q}(k) \tilde{A}_{i}^{\mathrm{T}}+\tilde{V}
$$

Lemma 4.1. $A_{e}$ is asymptotically stable if and only if

$$
\mathscr{A} \triangleq \tilde{A} \otimes \tilde{A}+\sum_{i=1}^{p} \tilde{A_{i}} \otimes \tilde{A}_{i}
$$

is asymptotically stable.

Proof. The result follows from properties of the Kronecker product applied to partitioned matrices. See [36] for details.

Hence $\mathscr{A}$ stable assures

$$
\tilde{Q} \triangleq \lim _{k \rightarrow \infty} \mathrm{E}\left[\tilde{x}(k) \tilde{x}^{\mathrm{T}}(k)\right]
$$

exists. Furthermore, $\tilde{Q}$ and its nonnegative-definite dual $\tilde{P}$ are the unique solutions of the modified Lyapunov equations

$$
\begin{aligned}
& \tilde{Q}=\tilde{A} \tilde{Q} \tilde{A}^{\mathrm{T}}+\sum_{i=1}^{p} \tilde{A_{i}} \tilde{Q} \tilde{A}_{i}^{\mathrm{T}}+\tilde{V}, \\
& \tilde{P}=\tilde{A}^{\mathrm{T}} \tilde{P} \tilde{A}+\sum_{i=1}^{p} \tilde{A}_{i}^{\mathrm{T}} \tilde{P} \tilde{A_{i}}+\tilde{R} .
\end{aligned}
$$

Partition $\left(n+n_{\mathrm{e}}\right) \times\left(n+n_{\mathrm{e}}\right) \tilde{Q}, \tilde{P}$ into $n \times n, n \times n_{\mathrm{e}}$, and $n_{\mathrm{e}} \times n_{\mathrm{e}}$ subblocks as

$$
\tilde{Q}=\left[\begin{array}{cc}
Q_{1} & Q_{12} \\
Q_{12}^{\mathrm{T}} & Q_{2}
\end{array}\right], \quad \tilde{P}=\left[\begin{array}{cc}
P_{1} & P_{12} \\
P_{12}^{\mathrm{T}} & P_{2}
\end{array}\right]
$$

and define the $n \times n$ nonnegative-definite matrices

$$
\begin{array}{ll}
Q \triangleq Q_{1}-Q_{12} Q_{2}^{-1} Q_{12}^{\mathrm{T}}, & \hat{\hat{Q}} \triangleq Q_{12} Q_{2}^{-1} Q_{12}^{\mathrm{T}}, \quad P \triangleq P_{1}-P_{12} P_{2}^{-1} P_{12}^{\mathrm{T}}, \quad \hat{\hat{P}} \triangleq P_{12} P_{2}^{-1} P_{12}^{\mathrm{T}}, \\
\hat{Q} \triangleq A \hat{\hat{Q}} A^{\mathrm{T}}+Q_{\mathrm{s}} V_{2 \mathrm{~s}}^{-1} Q_{\mathrm{s}}^{\mathrm{T}}, & \hat{P} \triangleq\left(A-Q_{\mathrm{s}} V_{2 \mathrm{~s}}^{-1} C\right)^{\mathrm{T}} \hat{\hat{P}}\left(A-Q_{\mathrm{s}} V_{2 \mathrm{~s}}^{-1} C\right)+\left(L-D_{\mathrm{e}} C\right)^{\mathrm{T}} R\left(L^{-}-D_{\mathrm{e}} C\right),
\end{array}
$$

where $\tau \hat{Q}^{\mathrm{T}}$ is replaced by $\hat{Q}$ in $Q_{s}$ and $V_{2 s}$ and the $n_{\mathrm{e}} \times n, n_{\mathrm{e}} \times n_{\mathrm{e}}, n_{\mathrm{e}} \times n$ matrices

$$
G \triangleq Q_{2}^{-1} Q_{12}^{\mathrm{T}}, \quad M \triangleq Q_{2} P_{2}, \quad \Gamma \triangleq-P_{2}^{-1} P_{12}^{\mathrm{T}} \text {. }
$$

To minimize (3.5) subject to the constraint (4.4), form the Lagrangian

$$
\mathscr{L}\left(A_{\mathrm{e}}, B_{\mathrm{e}}, C_{\mathrm{e}}, D_{\mathrm{e}}, \tilde{Q}, \tilde{P}, \lambda\right) \triangleq \operatorname{tr}\left[\lambda J\left(A_{\mathrm{e}}, B_{\mathrm{e}}, C_{\mathrm{e}}, D_{\mathrm{e}}\right)+\left(\tilde{A} \tilde{Q} \tilde{A}^{\mathrm{T}}+\sum_{i=1}^{p} \tilde{A_{i}} \tilde{A^{\mathrm{A}}} \tilde{A}_{i}^{\mathrm{T}}+\tilde{V}-\tilde{Q}\right) \tilde{P}\right],
$$

where the Lagrange multipliers $\lambda \geqslant 0$ and $\tilde{P} \in \mathbb{R}^{\left(n+n_{\mathrm{e}}\right) \times\left(n+n_{\mathrm{e}}\right)}$ are not both zero. Setting $\partial \mathscr{L} / \partial \tilde{Q}=0, \lambda=0$ 
implies $\tilde{P}=0$ since $\left(A_{\mathrm{e}}, B_{\mathrm{c}}, C_{\mathrm{e}}, D_{\mathrm{c}}\right) \in \mathscr{S}^{+}$. Hence, without loss of generality set $\lambda=1$. Thus the stationarity conditions are given by

$$
\begin{aligned}
& \frac{\partial \mathscr{L}}{\partial \tilde{P}}=\tilde{A} \tilde{Q} \tilde{A}^{\mathrm{T}}+\sum_{i=1}^{p} \tilde{A}_{i} \tilde{Q} \tilde{A}_{i}^{\mathrm{T}}+\tilde{V}-\tilde{Q}=0, \\
& \frac{\partial \mathscr{L}}{\partial \tilde{Q}}=\tilde{A}^{\mathrm{T}} \tilde{P} \tilde{A}+\sum_{i=1}^{p} \tilde{A}_{i}^{\mathrm{T}} \tilde{P} \tilde{A}_{i}+\tilde{R}-\tilde{P}=0, \\
& \frac{\partial \mathscr{L}}{\partial A_{\mathrm{e}}}=P_{12}^{\mathrm{T}} A Q_{12}+P_{2} B_{\mathrm{e}} C Q_{12}+P_{2} A_{\mathrm{e}} Q_{2}=0, \\
& \frac{\partial \mathscr{L}}{\partial B_{\mathrm{e}}}=P_{12}^{\mathrm{T}} Q_{s}+P_{2} B_{\mathrm{e}} V_{2 \mathrm{~s}}=0 \\
& \frac{\partial \mathscr{L}}{\partial C_{\mathrm{e}}}=-R L Q_{12}+R D_{\mathrm{e}} C Q_{12}+R C_{\mathrm{e}} Q_{2}=0, \\
& \frac{\partial \mathscr{L}}{\partial D_{\mathrm{e}}}=D_{\mathrm{e}} V_{2 \mathrm{~s}}-L Q C^{\mathrm{T}}=0 .
\end{aligned}
$$

Expanding (4.6) and (4.7) yields

$$
\begin{aligned}
& A(Q+\hat{\hat{Q}}) A^{\mathrm{T}}+\sum_{i=1}^{p} A_{i}(Q+\hat{\hat{Q}}) A_{i}^{\mathrm{T}}+V_{1}-Q-\hat{\hat{Q}}=0, \\
& {\left[A \hat{\hat{Q}} A^{\mathrm{T}}+Q_{\mathrm{s}} V_{2 s}^{-1} Q_{s}^{\mathrm{T}}-\hat{\hat{Q}}\right] \Gamma^{\mathrm{T}}=0,} \\
& \Gamma\left[A \hat{\hat{Q}} A^{\mathrm{T}}+Q_{s} V_{2 s}^{-1} Q_{s}^{\mathrm{T}}-\hat{\hat{Q}}\right] \Gamma^{\mathrm{T}}=0, \\
& {\left[\left(A-Q_{\mathrm{s}} V_{2 s}^{-1} C\right)^{\mathrm{T}} \hat{\hat{P}}\left(A-Q_{s} V_{2 s}^{-1} C\right)+\left(L-D_{\mathrm{e}} C\right)^{\mathrm{T}} R\left(L-D_{\mathrm{e}} C\right)-\hat{\hat{P}}\right] G^{\mathrm{T}}=0,} \\
& G\left[\left(A-Q_{\mathrm{s}} V_{2 s}^{-1} C\right)^{\mathrm{T}} \hat{\hat{P}}\left(A-Q_{\mathrm{s}} V_{2 s}^{-1} C\right)+\left(L-D_{\mathrm{e}} C\right)^{\mathrm{T}} R\left(L-D_{\mathrm{e}} C\right)-\hat{\hat{P}}\right] G^{\mathrm{T}}=0 .
\end{aligned}
$$

Note that the $(1,1)$ subblock of equation (4.7) which characterizes $P_{1}$ has been omitted from the above equations since the estimator gains are independent of $P_{1}$.

Using (4.8)-(4.11) we obtain (3.10)-(3.13). Using (4.12) $\left.+G^{\mathrm{T}} \Gamma(4.13) G-(4.13) G-((4.13) G)\right)^{\mathrm{T}}$ and $\left.G^{\mathrm{T}} \Gamma(4.13) G-(4.13) G-((4.13) G)\right)^{\mathrm{T}}+(4.13)-(4.13)$ yields $(3.14)$ and $(3.15)$. Using $\Gamma^{\mathrm{T}} G(4.15) \Gamma-(4.15) \Gamma$ $-((4.15) \Gamma))^{\mathrm{T}}+(4.15)-(4.15)$ yields $(3.16)$. Finally, $\Gamma(4.13)-(4.14)$ or $G(4.15)-(4.16)$ yields $\Gamma G^{\mathrm{T}}=I_{n_{\mathrm{e}}}$.

\section{References}

[1] D.S. Bernstein and D.C. Hyland, The optimal projection equations for reduced-order state estimation, IEEE Trans. Automat. Control 30 (1985) 583-585.

[2] D.S. Bernstein, L.D. Davis, S.W. Greeley and D.C. Hyland, The optimal projection equations for reduced-order, discrete-time modelling, estimation and control, Proc. 24th IEEE Conf. Decision and Control, Fort Lauderdale, FL (Dec. 1985) pp. 573-578.

[3] D.S. Bernstein, L.D. Davis and D.C. Hyland, The optimal projection equations for reduced-order, discrete-time modelling, estimation and control, J. Guid. Control Dyn. 9 (1986) 288-293.

[4] C.S. Sims and J.L. Melsa, A survey of specific optimal techniques in control and estimation, Internat. J. Control 12 (1971) 299-308.

[5] T.E. Fortman and D. Williams, Design of low-order observers for linear feedback control laws, IEEE Trans. Automat. Control 17 (1972) 301-308.

[6] C.S. Sims, An algorithm for estimating a portion of a state vector, IEEE Trans. Automat. Control 19 (1974) $391-393$.

[7] R.B. Asher, K.D. Herring and J.C. Ryles, Bias variance and estimation error in reduced-order filters, Automatica 12 (1976) $289-600$. 
[8] J.I. Galdos and D.E. Gustafson, Information and distortion in reduced-order filter design, IEEE Trans. Inform. Theory 23 (1977) 183-194.

[9] F.W. Fairman, Reduced-order state estimators for discrete-time stochastic systems, IEEE Trans. Automat. Control 22 (1977) 673-675.

[10] F.W. Fairman, On stochastic observer estimators for continuous-time systems, IEEE Trans. Automat. Control 22 (1977) 874-876.

[11] C.S. Sims and R.B. Asher, Optimal and suboptimal results in full and reduced-order linear filtering, IEEE Trans. Automat. Control 23 (1978) 469-472.

[12] C.S. Sims and L.G. Stotts, Linear discrete reduced-order filtering, Proc. IEEE Conf. Decision and Control (1979) pp. 1172-1177.

[13] D.A. Wilson and R.N. Mishra, Design of low order estimators using reduced models, Internat. J. Control 23 (1979) $447-456$.

[14] F.W. Fairman and R.D. Gupta, Design of multifunctional reduced-order observers, Internat. J. Systems Sci. 11 (1980) 1083-1094.

[15] U.V. Dombrovskii, Method of synthesizing suboptimal filters of reduced order for digital linear dynamic systems, Automat. Remote Control 43 (1982) 1483-1489.

[16] T. Hinamoto and F.W. Fairman, Reduced order observer design for a linear map of the state, J. Franklin Inst. 314 (1982) 95-108.

[17] C.S. Sims, Reduced-order modelling and filtering, in: C.T. Leondes, Ed., Control and Dynamic Systems Vol. 18 (Academic Press, New York, 1982) pp. 55-103.

[18] P.J. McLane, Optimal linear filtering for linear systems with state-dependent noise, Internat. J. Control 10 (1969) $41-51$.

[19] Y. Sunahara and K. Yamashita, An approximate method of estimation for non-linear dynamical systems with state-dependent noise, Internat. J. Control 11 (1970) 957-972.

[20] D.E. Gustafson and J.L. Speyer, Linear minimum variance filters applied to carrier tracking, IEEE Trans. Automat. Control 21 (1976) 65-73.

[21] R.B. Asher and C.S. Sims, Reduced-order filtering with state dependent noise, Proc. Joint Amer. Control Conf. (1978).

[22] C.S. Sims, Discrete reduced-order filtering with state-dependent noise, Proc. Joint Autom. Control Conf. (1980).

[23] H.V. Panossian and C.T. Leondes, Observers for optimal estimation of the state of linear stochastic discrete systems, Internat. $J$. Control 37 (1983) 645-655.

[24] M.J. Grimble, Wiener and Kalman filters for systems with random parameters, IEEE Trans. Automat. Control 29 (1984) $552-554$.

[25] H. Heffes, The effect of erroneous models on the Kalman filter response, IEEE Trans. Automat. Control 11 (1966) $541-543$.

[26] J.A. D'Appolito and C.E. Hutchinson, Low sensitivity filter for state estimation in the presence of large parameter uncertainties, IEEE Trans. Automat. Control 14 (1969) 310-312.

[27] D.G. Lainotis and F.L. Sims, Sensitivity analysis of discrete Kalman filters, Internat. J. Control 12 (1970) 657-669.

[28] J.M. Morris, The Kalman filter: A robust estimator for some classes of linear quadratic problems, IEEE Trans. Inform. Theory 22 (1976) 526-534.

[29] C.J. Masreliez and R.D. Martin, Robust Bayesian estimation for the linear model and robustifying the Kalman filter, IEEE Trans. Automat. Control 22 (1977) 361-371.

[30] M. Toda and R.V. Patel, Bounds on estimation error of discrete-time filters under modelling uncertainty, IEEE Trans. Automat. Control 25 (1980) 1115-1121.

[31] R.T. Stefani, Reducing the sensitivity to parameter variations of a minimum-order reduced-order observer, Internat. J. Control 35 (1982) 983-995.

[32] D.S. Bernstein and D.C. Hyland, The optimal projection/maximum entropy approach to designing low-order, robust controllers for flexible structures, Proc. 24th IEEE Conf. Decision and Control, Fort Lauderdale, FL (Dec. 1985) pp. 745-752.

[33] D.S. Bernstein, L.D. Davis, S.W. Greeley and D.C. Hyland, Numerical solution of the optimal projection/maximum entropy design equations for low-order, robust controller design, Proc. 24th IEEE Conf. Decision and Control, Fort Lauderdale, FL (Dec. 1985) pp. 1795-1798.

[34] D.S. Bernstein and S.W. Greeley, Robust controller synthesis using the maximum entropy design equations, IEEE Trans. Automat. Control 31 (1986) 362-364.

[35] D.S. Bernstein and S.W. Greeley, Robust output-feedback stabilization: Deterministic and stochastic perspectives, Proc. Amer. Control Conf., Seattle, WA (June 1986) pp. 1818-1826.

[36] D.S. Bernstein and D.C. Hyland, The optimal projection equations for reduced-order modelling, estimation and control of linear systems with'Stratonovich multiplicative white noise, submitted.

[37] D.S. Bernstein, Robust static and dynamic output-feedback stabilization: Deterministic and stochastic perspectives, submitted.

[38] D.S: Bernstein, Robust stability and performance via the extended optimal projection equations for fixed-order dynamic compensation, submitted.

[39] J.W. Brewer, Kronecker products and matrix calculus in System theory, IEEE Trans. Circuits and Systems 25 (1978) $772-781$.

[40] C.R. Rao and S.K. Mitra, Generalized Inverse of Matrices and Its Applications (John Wiley and Sons, New York, 1971). 